\title{
Panorama Nacional da Pesquisa Sobre Avaliação Neuropsicológica de Linguagem
}

A national view on the research concerning the neuropsychological evaluation of language

Adriana Jung Serafini, Rochele Paz Fonseca, Denise Ruschel Bandeira \& Maria Alice de Mattos Pimenta Parente

Universidade Federal do Rio Grande do Sul 
Resumo: Apesar da grande difusão de instrumentos internacionais de avaliação da linguagem no contexto neuropsicológico, no Brasil, verifica-se uma importante demanda de técnicas adequadas à realidade sócio-lingüístico-cultural nas áreas clínica e de pesquisa. O objetivo deste estudo é apresentar um panorama nacional das pesquisas que envolvem a avaliação de linguagem no contexto da neuropsicologia. Para tanto, foram realizadas buscas relativas a estudos brasileiros nos bancos de Teses e Dissertações da CAPES, IBICT Teses e Index Psi Teses, e nos bancos de periódicos LILACS, SciELO e Index Psi Periódicos Técnico-Científicos. Como resultado, verificouse um número reduzido de pesquisas sobre avaliação neuropsicológica da linguagem, sendo que a maior parte das publicações foi encontrada em periódicos de áreas médicas. Poucos estudos faziam referência a algum tipo de instrumento, e raros foram os que visaram a fins psicométricos. Entretanto, observou-se um aumento do número de artigos científicos sobre avaliação de linguagem no contexto neuropsicológico, predominantemente nos últimos seis anos.

Palavras-chave: Neuropsicologia. Avaliação neuropsicológica. Linguagem. Psicometria.

Abstract: Although there is a great variety of international tests on language evaluation in the neuropsychological context, there's a significant demand of techniques adapted to the social, linguistic and cultural reality of Brazil in the clinical and research domains. This study aims to present a national view on research on language assessment in the context of neuropsychology. To accomplish this, a search for Brazilian studies in the following thesis and dissertation databases was performed: CAPES, IBICT Teses and Index Psi Teses, and in LILACS, SciELO and Index Psi Science Journals. As a result, little research concerning the neuropsychological evaluation of language was verified, and the majority of papers was published in medical journals. Not many made reference to some type of instrument, and those focused on psychometrics were rare. However, an increase of papers on the neuropsychological evaluation of language is observed, mainly during the last six years.

Keywords: Neuropsychology. Neuropsychological evaluation. Language. Psychometrics.

A Neuropsicologia é tradicionalmente definida como a área de estudo das relações entre o cérebro e o comportamento (Gil, 2005; Hebben \& Milberg, 2002; Lezak, Howieson \& Loring, 2004). De acordo com Luria (1981), essa ciência tem como objetivo investigar o papel dos sistemas cerebrais de cada indivíduo nas formas complexas de atividade mental. Seu caráter interdisciplinar é destacado por Rao (1996), que ressalta o envolvimento de especialistas da Neurologia, Psiquiatria, Psicologia, Fonoaudiologia e Lingüística, entre outros. A noção de interdisciplinaridade na Neuropsicologia surge com as duas Grandes Guerras Mundiais, sendo enfatizada nos trabalhos clássicos de Alajouanine, Ombredane e Durand (1939) e Alajouanine e Lhermitte (1960).
No que concerne à sua história, o marco inicial da neuropsicologia moderna corresponde aos estudos de caso do médico neurologista Paul Broca, apresentados em 1861, que associou um quadro de dificuldades de expressão lingüística a uma lesão no lobo frontal do hemisfério cerebral esquerdo, área que veio a receber seu nome. Graças a essa descoberta, primeiramente, a neuropsicologia nasceu com o estudo da afasia, ou seja, o distúrbio da forma, conteúdo ou uso da linguagem após um acometimento neurológico - área reconhecida mais tarde como afasiologia (Brookshire, 2003). A palavra neuropsicologia foi usada pela primeira vez por Osler, em 1913 (Benedet, 1995; Siksou, 2005).

Uma das aplicações clínicas da Neuropsicologia é o processo de avaliação neuropsicológica. 
A temática

central do

presente estudo

é a avaliação

neuropsicológica

da função

linguagem, que é considerada por

Harley (2001) um

sistema complexo

de símbolos e

regras que permite

a comunicação,

com o

envolvimento de

habilidades de

compreensão e expressão.
Essa avaliação corresponde ao método de investigação das funções cerebrais a partir de seu produto comportamental (Mäder, 2002) e baseia-se nos conhecimentos de três grandes áreas: Medicina (neurologia, Psiquiatria, Pediatria, Geriatria, entre outras), Psicologia (Psicologia Cognitiva, Psicologia clínica, psicopatologia e Psicometria Clássica) e Lingüística (pragmática e semântica, entre outras). Além disso, os conhecimentos de Psicolingüística, provenientes da Psicologia e da Lingüística, são fundamentais para o corpo teórico-metodológico da avaliação neuropsicológica (Manning, 2005; Siksou, 2005).

A avaliação neuropsicológica apresenta como finalidades a averiguação da ocorrência de distúrbios cognitivos relacionados à lesão cerebral, à determinação da magnitude dessa lesão, ao estabelecimento da capacidade do indivíduo de retornar ao padrão de funcionamento pré-mórbido e à elaboração de um programa específico de reabilitação para delimitar as modificações ambientais necessárias a melhor adaptação da pessoa às suas novas condições cognitivas (Ardila \& Ostrosky-Solís, 1993; Lezak, Lezak et al., ; Mccormick \& Lezak, 2005; Sohlberg \& Mateer, 2001). Constata-se, então, que, para o alcance desses objetivos, um instrumento de avaliação deve estar norteado por diferentes abordagens teórico-metodológicas, tais como a Psicologia cognitiva, a psicolingüística e a psicometria, e por um método interdisciplinar oriundo da neurologia, da Psicologia e da Lingüística.

Quanto aos procedimentos de avaliação neuropsicológica, ressalta-se que o uso de instrumentos complementa a avaliação clínica. $\mathrm{Na}$ literatura, destacam-se duas classes de instrumentos de avaliação neuropsicológica: (1) instrumentos de exploração flexível e (2) instrumentos de exploração objetiva e sistemática. A primeira categoria é representada por tarefas neuropsicológicas, que são ferramentas de avaliação adaptadas e construídas para cada caso, sem uma padronização de grupo. Mirsky (1989) sugere, por exemplo, que, para a avaliação da atenção focalizada, sejam utilizados o Trail Making Test, uma tarefa de cancelamento, e o Stroop Test. A segunda categoria é composta por baterias neuropsicológicas ou baterias para cada função padronizadas, com base, principalmente, em critérios de legitimação psicométrica de instrumentos: normatização, fidedignidade, sensibilidade, especificidade e validação. A bateria neuropsicológica LuriaNebraska é um exemplo dessa categoria (Kane, 1991; Lezak et al., 2004; Strauss, Sherman \& Spreen, 2006). Apesar da importância das baterias padronizadas no processo moderno de avaliação neuropsicológica, esta se caracteriza tradicionalmente pela construção de tarefas, ou seja, privilegia em sua rotina o uso de procedimentos não padronizados.

As funções cognitivas geralmente avaliadas são atenção, percepção, orientação temporal e viso-espacial, linguagem (oral e escrita), raciocínio, memória, cálculo, praxias e funções executivas (Andrade, 2002; Casanova, 1987; Lezak et al. 2004, Strauss et al., 2006). A temática central do presente estudo é a avaliação neuropsicológica da função linguagem, que é considerada por Harley (2001) um sistema complexo de símbolos e regras que permite a comunicação, com o envolvimento de habilidades de compreensão e expressão. No contexto neuropsicológico, a avaliação da linguagem nasceu com o exame da afasia, junto ao surgimento da neuropsicologia. É necessária em quadros de acometimentos neurológicos, principalmente em quadros de lesões vasculares, traumatismos crânio-encefálicos, epilepsias e demências. Além dessa demanda neuropsicológica clínica clássica, a linguagem também é examinada na neuropsicologia cognitiva, área interessada na análise de funções e processos mantidos, assim como dos déficits cognitivos, para a 
"A avaliação

neuropsicológica

da linguagem

diferencia-se

da avaliação

da linguagem

em geral

(avaliação não-

neuropsicológica

da linguagem)

por ter como

objetivo distinguir

habilidades

lingüísticas

preservadas e

deficitárias após

acometimentos

neurológicos"

Lezak et al. melhor compreensão da organização do comportamento normal (Seron, 1997). Na neuropsicologia cognitiva, a linguagem é avaliada em indivíduos de diferentes fases do desenvolvimento humano, e pode ser examinada individualmente devido a um quadro de distúrbio compreensivo e/ou expressivo apenas, ou como parte de uma bateria de avaliação de todas as funções neuropsicológicas. A avaliação neuropsicológica da linguagem diferencia-se da avaliação da linguagem em geral (avaliação não-neuropsicológica da linguagem) por ter como objetivo distinguir habilidades lingüísticas preservadas e deficitárias após acometimentos neurológicos (Lezak et al., 2004; Siksou, 2005).

Apesar da grande difusão de instrumentos internacionais de avaliação da linguagem no contexto neuropsicológico, no Brasil, verifica-se, ainda, uma importante demanda de instrumentos de avaliação adequados às diversas realidades sociolingüístico-culturais nas áreas clínica e de pesquisa. Na tentativa de melhor caracterizar essa demanda, torna-se importante averiguar como a avaliação neuropsicológica de linguagem está sendo pesquisada nacionalmente. $\mathrm{O}$ presente estudo tem por objetivo apresentar o panorama nacional de pesquisas que envolvem a avaliação de linguagem no contexto da neuropsicologia. Para tanto, as seguintes questões serão respondidas:

- Quantas teses encontradas em bancos nacionais de teses abordam a temática avaliação de linguagem e, mais especificamente, avaliação de linguagem no contexto da neuropsicologia?

- Quantos artigos científicos indexados em bases nacionais abordam a temática avaliação de linguagem e, mais especificamente, avaliação de linguagem no contexto da neuropsicologia?

- Em quais áreas os artigos científicos que abordam a avaliação de linguagem no contexto da neuropsicologia são publicados?

- Há um crescimento do número de publicações sobre avaliação de linguagem no contexto da neuropsicologia no decorrer dos anos?

- Quantas publicações nacionais que envolvem instrumentos de avaliação da linguagem se relacionam ao contexto da neuropsicologia e/ou a fins psicométricos?

- Quantas publicações nacionais são encontradas sobre cada instrumento de avaliação da linguagem utilizado na pesquisa em neuropsicologia?

As respostas para essas questões foram buscadas a partir de uma revisão sistemática da literatura em bases eletrônicas de dados (resumos de dissertações, teses e artigos científicos). Esse método caracteriza-se pela seleção de estudos indexados nas referidas bases a partir de determinadas palavras-chave. Na neuropsicologia, esse tipo de estudo vem se tornando cada vez mais freqüente. Como exemplos, mencionam-se dois estudos na temática avaliação neuropsicológica: o estudo de Argimon, Timm, Rigoni e Oliveira (2005), em que os autores buscaram abstracts indexados de investigações sobre os instrumentos que avaliam a memória em idosos com as palavras-chave assessment of memory (avaliação da memória) e elderly (idosos), e o estudo de Vasconcelos, Brucki e Bueno (2007), no qual se procurou identificar as ferramentas de avaliação cognitiva e funcional mais utilizadas no diagnóstico de demência no Brasil, através de uma busca em bancos de dados com as palavras-chave dementia (demência) e Alzheimer. No âmbito da reabilitação neuropsicológica, outro estudo também ilustra esse tipo de revisão sistemática da literatura: Cicerone et al. (2005), com o objetivo de promover uma atualização dos procedimentos de 
reabilitação cognitiva baseados em evidências em populações de traumatizados cranianos e indivíduos que sofreram acidente vascular encefálico, efetuaram uma busca nas bases de dados com termos referentes a cada função neuropsicológica, tais como attention (atenção), associados às palavras-chave rehabilitation (reabilitação), remediation (remediação) e training (treinamento).

\section{Método}

Para se responder à primeira questão de pesquisa, realizou-se uma busca sistemática dos resumos nas bases nacionais de dissertações e Teses, a saber: Index Psi Teses, IBICT Teses (Biblioteca Digital de teses e Dissertações - IBICT) e Banco de teses da CAPES. Nessas bases, o levantamento abrangeu estudos desde o começo da indexação, ou seja, desde o início da base para Index Psi Teses e IBICT Teses (data não mencionada nos respectivos sites) e desde 1987 para o Banco de Teses da CAPES. Utilizaram-se as seguintes palavras-chave em português: "avaliação AND linguagem", "instrumento AND linguagem" e "teste AND linguagem". Os critérios de inclusão dos estudos encontrados pela busca inicial com as palavras-chave acima foram os seguintes: a) consistir em uma dissertação ou tese que aborde a avaliação de linguagem que represente o exame da função lingüística em seres humanos, e b) ser um estudo de caso ou um estudo empírico. Com o objetivo de determinar a quantidade de dissertações e teses que abordavam a temática avaliação de linguagem no contexto específico da neuropsicologia, os resumos obtidos através da busca descrita nos critérios "a" e " $b$ " foram novamente analisados. Nessa re-análise, buscou-se verificar aqueles que mencionavam tarefas ou testes de linguagem utilizados com objetivos condizentes com os pressupostos da avaliação neuropsicológica, conforme revisados na seção Introdução. Procurouse, por exemplo, averiguar se os estudos citavam o termo avaliação neuropsicológica ou instrumentos específicos dessa área (como baterias neuropsicológicas), se estavam relacionados à avaliação de linguagem em quadros neurológicos, tais como epilepsia, traumatismo crânio-encefálico, acidentes vasculares encefálicos, tumores, entre outros, e/ou neuropsicológicos, tais como afasias, dislexias, disartrias, dispraxias, etc. Além disso, também se verificou se os estudos correspondiam a pesquisas na área de neuropsicologia cognitiva, como a investigação do processamento cognitivolingüístico em populações neurologicamente preservadas, com o intuito de se obter um melhor entendimento do processamento da linguagem e das funções cognitivas correlatas.

Quanto à segunda questão de pesquisa, foram consultadas as bases de indexação SciELO, LILACS e Index Psi Periódicos Técnico-Científicos. Quanto ao período do levantamento, foram levantados resumos desde o surgimento das referidas bases eletrônicas, isto é, desde 1949, na base Index Psi Periódicos Técnico Científicos, desde 1982, na base LILACS, e, desde 1997, na base SciELO. As palavras-chave foram aquelas lançadas na busca da primeira questão de pesquisa. Para se encontrar estudos sobre a avaliação de linguagem no contexto da neuropsicologia, foram realizados os mesmos procedimentos usados na busca nas bases de dados de dissertações e teses.

Com objetivo de esclarecer a terceira e a quarta questão, quantificou-se o número de resumos dos estudos encontrados no contexto da neuropsicologia por área do periódico em que foram publicados e as datas de publicação dos mesmos, respectivamente. A quinta questão foi respondida através da análise de cada um dos resumos. Para aquelas pesquisas que citaram a utilização de instrumentos para avaliação de linguagem, averiguou-se primeiramente se esses estudos estavam 
relacionados à área da neuropsicologia (os critérios utilizados para tal já foram citados no método para responder à questão 1). Posteriormente, foi investigado se os estudos com instrumentos da área de linguagem geral, ou especificamente do contexto neuropsicológico, tinham por objetivo traduzir, validar, adaptar ou realizar estudos sobre a sensibilidade, a especificidade, a precisão e/ou a normatização de tais técnicas. Em relação à sexta questão, foram listados todos os instrumentos utilizados para avaliação de linguagem em cada uma das pesquisas do campo da neuropsicologia, sendo que alguns estudos citaram mais de uma técnica.

Para responder a todas as questões de pesquisa, as buscas em bases de dados foram realizadas no mês de junho de 2006. Os dados foram analisados a partir da estatística descritiva. Somente foram analisados e quantificados aqueles estudos que apresentaram seus resumos indexados nas bases de dados, escritos na língua portuguesa em sua variedade brasileira. Em relação às bases de teses e dissertações, no Banco de Teses CAPES, foram analisados apenas os resumos de teses de doutorado, excluindo-se as dissertações de mestrado, já que, para uma primeira análise, teriam que ser avaliados um total de 1.914 resumos de dissertações (número que foi obtido a partir da busca por palavras-chave).

Para a quantificação de resumos de teses e dissertações e de artigos científicos, adotaramse os seguintes procedimentos: 1) a partir da base de dados que apresentou o maior número de estudos (no caso das teses e dissertações, o Banco de Teses da CAPES, e dos artigos, a base LILACS), excluíram-se das demais aqueles resumos que apareceram repetidos nos resultados das buscas. 2) Como nas duas formas de avaliação (por dissertações e teses e por artigos) haviam sido analisados três bancos de dados, o mesmo procedimento realizado na etapa 1 teve que ser replicado na segunda base de dados mais numerosa e na terceira, excluindo-se desta última aqueles estudos que se repetiram entre elas.

Para ambos os tipos de bancos de dados (dissertações e teses e periódicos), também foram excluídos da quantificação os estudos que se repetiram nas buscas pelas diferentes palavras-chave ("avaliação AND linguagem", "instrumentos AND linguagem" e "teste AND linguagem"), utilizando-se os mesmos critérios para a análise anterior. Os resultados da busca pela palavra-chave "avaliação AND linguagem" serviu de referência por ter sido aquela que apresentou maior número de pesquisas, excluindo-se das demais os estudos que apareceram repetidos. Posteriormente, uma nova triagem foi realizada entre a segunda busca com maior número de resultados ("instrumentos AND linguagem") e a terceira ("testes AND linguagem").

No que se refere à categorização dos estudos avaliados, o primeiro procedimento foi listar as revistas em que os estudos de avaliação de linguagem no campo da neuropsicologia foram publicados. Após essa etapa, investigaramse quais eram os órgãos editorais de cada uma dessas revistas. Quando esse item não constava, ou não ficava claro através dos dados da revista, avaliou-se a área de atuação dos editores e/ou a área ou público ao qual a revista relatava dirigir-se.

\section{Resultados}

Nesta seção, as questões de pesquisa são respondidas mediante uma análise descritiva dos dados da literatura. Na Tabela 1, visualizase a quantidade de dissertações de mestrado e teses de doutorado encontradas nas bases de dados CAPES, IBICT Teses e Index Psi Teses que abordam a temática avaliação de linguagem e avaliação de linguagem no contexto neuropsicológico. 
Tabela 1: Quantidade de dissertações e teses com a temática avaliação da linguagem inseridas ou não no contexto neuropsicológico

\begin{tabular}{lll}
\hline Banco de teses & Avaliação da linguagem & $\begin{array}{l}\text { Avaliação da linguagem no contexto } \\
\text { neuropsicológico }\end{array}$ \\
\hline CAPES & 68 & 19 \\
IBICT Teses & 18 & 03 \\
Index Psi Teses & 02 & 00 \\
Total & 88 & 22 \\
\hline
\end{tabular}

A observação da Tabela 1 permite constatar que somente $25 \%$ dos 88 abstracts da quantificação final de teses focalizavam a avaliação da linguagem no contexto da neuropsicologia. Em complementaridade a essa análise, na Tabela 2, apresentam-se dados referentes ao número de artigos científicos encontrados cujo foco se centra na avaliação de linguagem e avaliação de linguagem no campo da neuropsicologia.

Tabela 2: Quantidade de artigos científicos com a temática avaliação de linguagem inseridos ou não no contexto neuropsicológico

\begin{tabular}{lll}
\hline Banco de Periódicos & Avaliação da linguagem & $\begin{array}{l}\text { Avaliação da linguagem no contexto } \\
\text { neuropsicológico }\end{array}$ \\
\hline LILACS & 81 & 16 \\
SciELO & 13 & 07 \\
Index Psi & 05 & 00 \\
Total & 99 & 23 \\
\hline
\end{tabular}

Do total de 99 resumos sobre a temática avaliação de linguagem, 23,24\% abordava especificamente a avaliação de linguagem no contexto da neuropsicologia. Nota-se, então, que somente cerca de um quarto das publicações sobre avaliação de linguagem, tanto em teses e dissertações como em artigos científicos, faz referência especificamente à avaliação neuropsicológica da linguagem. Os resultados da análise da quantidade de artigos científicos sobre o tópico avaliação da linguagem no contexto da neuropsicologia por área de publicação podem ser visualizados na Tabela 3.

Tabela 3: Quantidade de artigos científicos sobre avaliação da linguagem no contexto neuropsicológico por área de publicação

\begin{tabular}{|c|c|c|c|}
\hline Áreas de publicação & Revistas * & Total por revista & $\begin{array}{l}\text { Número de } \\
\text { publicações }\end{array}$ \\
\hline Neuropsiquiatria & Arquivos de neuropsiquiatria & 11 & 11 \\
\hline \multirow[t]{2}{*}{ Fonoaudiologia } & Pró-fono & 03 & 04 \\
\hline & Distúrbios da comunicação & 01 & \\
\hline \multirow[t]{2}{*}{ Psiquiatria } & Revista Brasileira de Psiquiatria & 01 & \\
\hline & Revista de Psiquiatria Clínica & 01 & 02 \\
\hline Saúde e educação & Temas sobre desenvolvimento & 02 & 02 \\
\hline Medicina geral & São Paulo Medical Journal & 01 & 01 \\
\hline Neurobiologia & Neurobiologia & 01 & 01 \\
\hline \multirow[t]{2}{*}{ Neurologia } & Journal of Epilepsy and Clinical & & \\
\hline & Neurophysiology & 01 & 01 \\
\hline Psicologia & Arquivos Brasileiros de Psicologia & 01 & 01 \\
\hline Total & & 23 & 23 \\
\hline
\end{tabular}


Os periódicos de áreas da Medicina, como Medicina geral, neurologia, psiquiatria, neuropsiquiatria e neurobiologia, foram os que mais publicaram artigos sobre avaliação de linguagem no contexto neuropsicológico (69,56\%), sendo seguidos pelos da fonoaudiologia, com 17,39\% das publicações, de um campo mais abrangente, definido pela própria revista como saúde e educação (8,69\%) e, por fim, da Psicologia, com 4,34\%. Em comparação com as demais revistas, o periódico Arquivos de Neuropsiquiatria foi aquele que englobou a maior porcentagem $(42,83 \%)$ das pesquisas sobre avaliação de linguagem no contexto neuropsicológico analisadas neste estudo.

Na Tabela 4, apresentam-se dados da análise da quantidade de publicações sobre a temática avaliação de linguagem no contexto da neuropsicologia por agrupamento de anos. Em geral, o período dos agrupamentos é de 5 anos.

Tabela 4: Quantidade de artigos sobre avaliação de linguagem no contexto neuropsicológico desde 1987

\begin{tabular}{ll}
\hline Ano & Número de artigos \\
\hline $1987-1990$ & 02 \\
$1991-1995$ & 03 \\
$1996-2000$ & 03 \\
$2001-2006$ & 15 \\
Total & 23 \\
\hline
\end{tabular}

Observa-se na Tabela 4 que houve um considerável crescimento de artigos científicos sobre avaliação de linguagem no contexto neuropsicológico. Salienta-se que 65,21\% dos artigos encontrados foram publicados nos últimos seis anos, enquanto 34,79\% das publicações restantes se mostraram distribuídas entre os anos 1987 e 2000. Na Tabela 5, são mostrados os resultados da quantificação feita dos estudos publicados em artigos científicos categorizados em neuropsicológicos e não neuropsicológicos, com e sem fins psicométricos.

Tabela 5: Quantidade de estudos por categoria de publicação

\begin{tabular}{ll}
\hline Categoria dos estudos & Número de estudos \\
\hline Não neuropsicológico, sem fins psicométricos & 35 \\
Neuropsicológico, sem fins psicométricos & 15 \\
Não neuropsicológico, com fins psicométricos & 05 \\
Neuropsicológico, com fins psicométricos & 02 \\
Total & 57 \\
\hline
\end{tabular}

Das 57 publicações encontradas na área de avaliação de linguagem que faziam referência a algum tipo de instrumento, mais da metade (70,17\%) englobava estudos não neuropsicológicos, sendo que somente 29,83\% dessas publicações foi categorizada como neuropsicológica. Além disso, quanto à finalidade psicométrica do estudo, apenas $12,50 \%$ das publicações possuíam fins psicométricos, tais como construção, tradução, adaptação, validação e fidedignidade de técnicas, entre outros procedimentos. Considerando-se os estudos categorizados como neuropsicológicos, só 3,50\% destes apresentava preocupação com a realização de procedimentos psicométricos.

Dessas 57 publicações, os 17 estudos que abordavam algum tipo de instrumento e que foram categorizados como neuropsicológicos serviram de base para que uma lista de instrumentos fosse 
elaborada. A quantificação de publicações a que se referia cada instrumento pode ser observada na Tabela 6.

Tabela 6: Quantidade de publicações de estudos neuropsicológicos por instrumento

Instrumentos
Número de publicações que mencionam

Teste de Nomeação de Boston 04

Teste Boston para Diagnóstico de Afasia 02

Teste de Fluência Verbal - Categoria Animais

02

Teste de Vocabulário por Imagens Peabody

02

Teste Token

ADAS-cog 01

Figuras temáticas yavas

01

Inventário de habilidades semânticas

01

Mini exame do estado mental

01

Neuropsi

01

Pediatric syntetic intellibility - PSI

01

Provas de reconhecimento semântico

01

Tarefa de fluência verbal

01

Teste de Competência de Leitura de Palavras - TCLP $\quad 01$

Teste de Linguagem Infantil ABFW

01

Teste de Triagem Denver - II

01

Teste M1-alpha

01

Total*

* O total dessa tabela corresponde ao número de publicações que citaram cada instrumento. Foi superior ao total de 17 publicações que fizeram referência a algum instrumento no contexto neuropsicológico, porque alguns estudos mencionavam mais de um instrumento.

Na Tabela 6, constata-se que, dos 17 instrumentos citados nos estudos publicados, o Teste de Nomeação de Boston foi o instrumento mais citado pelas pesquisas que abordavam algum instrumento de avaliação da linguagem no contexto neuropsicológico, aparecendo em 16,67\% das pesquisas. Na seqüência, os instrumentos Teste de Fluência Verbal - Categoria Animais, Teste de Vocabulário por Imagens Peabody e Teste Token foram citados por 8,34\% dos estudos. Os demais instrumentos foram mencionados por apenas $4,17 \%$ das investigações.

\section{Discussão}

O objetivo deste estudo foi apresentar um panorama nacional de pesquisas relativo à avaliação de linguagem no contexto da neuropsicologia. De modo geral, pode-se constatar que, apesar de ainda haver poucos estudos que envolvem essa temática, as pesquisas sobre avaliação neuropsicológica da linguagem vêm crescendo em quantidade, principalmente na área de publicação das ciências médicas.

No que diz respeito à quantidade de teses e de artigos científicos que abordam a temática avaliação de linguagem em geral e avaliação de linguagem no contexto da neuropsicologia, 
Provavelmente, a pesquisa nacional sobre essa avaliação tende a seguir o mesmo rumo de difusão das publicações internacionais. são encontrados mais estudos sobre a primeira. Esperava-se encontrar mais pesquisas sobre avaliação da linguagem com fins neuropsicológicos, uma vez que a investigação dos correlatos neurais dessa função fundou a ciência neuropsicológica (Benedet, 1995; Manning, 2005; Siksou, 2005). Internacionalmente, o processo de avaliação neuropsicológica da função linguagem é largamente difundido, tanto em pesquisas com baterias neuropsicológicas de avaliação de todas as funções cognitivas (Darvesh, Leach, Black, Kaplan, \& Freedman, 2005; Larson, Kirschner, Bode, Heinemanm, \& Goodman, 2005) como em investigações com instrumentos específicos de linguagem (Gonzáles, Mungas, \& Haan, 2005; Kalbe, Reinhold, Brand, Markowitsch, \& Kessler, 2005). Provavelmente, a pesquisa nacional sobre essa avaliação tende a seguir o mesmo rumo de difusão das publicações internacionais.

Essa reduzida quantidade de abstracts encontrada sobre avaliação neuropsicológica da linguagem pode estar relacionada à seleção restrita de palavras-chave no presente estudo. Os termos escolhidos foram mais amplos para possibilitar uma seleção pessoal a posteriori. No entanto, o termo "linguagem" pode ter sido muito geral para encontrar dissertações, teses e artigos sobre essa temática, já que os pesquisadores dessa área podem estar utilizando termos mais específicos do estudo de linguagem, tais como palavras referentes aos níveis lingüísticos, expressão ou produção e recepção ou compreensão, ou ainda relacionadas aos componentes da linguagem - fonologia, morfologia, sintaxe, semântica, pragmática e prosódia, por exemplo. Além disso, também pode ter havido problemas com os indexadores, que teriam limitado a busca pelas palavras-chave selecionadas, sem gerar todos os estudos que realmente investigaram a avaliação neuropsicológica da linguagem. Por fim, outro fator que pode ter colaborado para a pequena quantidade de resumos encontrada sobre o tema estudado diz respeito à não inclusão de dissertações de mestrado oriundas do Banco de Teses da CAPES.

As investigações encontradas sobre a temática em pauta estão concentradas predominantemente em periódicos nacionais da área da Medicina. Esse dado era esperado, tendo em vista que foram médicos neurologistas que fundaram a neuropsicologia ao estudar a base cerebral da linguagem nas afasias, com os estudos de Broca e de Wernicke sobre afasia de expressão e de compreensão, respectivamente, e nas dislexias - dificuldades de processamento da linguagem escrita - com o estudo de Déjerine (Manning, 2005). Além disso, os primeiros instrumentos publicados também foram elaborados por médicos neurologistas (Hebben \& Milberg, 2002). Após os periódicos da área médica, aqueles que mais publicam artigos sobre o exame da linguagem no contexto neuropsicológico foram os de fonoaudiologia, área do conhecimento que tem como um de seus focos a função lingüística e é muito influenciada pelos conhecimentos da afasiologia, atualmente conhecida como neuropsicologia (Brookshire, 2003).

Embora haja toda uma fundamentação histórico-epistemológica para a maior quantidade de publicações sobre avaliação neuropsicológica da linguagem em periódicos nacionais de Medicina, principalmente de neuropsiquiatria (Alchieri, 2003) e de fonoaudiologia, é surpreendente a descoberta que a Psicologia é a área que menos comunica estudos sobre o tópico em questão para a sua comunidade científica. A 
surpresa reside pelo fato de recentemente o Conselho Federal de Psicologia ter reconhecido a neuropsicologia como uma das especialidades do psicólogo Resolução no 2/2004 (Conselho Federal de Psicologia, 2004). Uma das explicações para a escassez de publicações sobre avaliação neuropsicológica da linguagem na Psicologia talvez seja o menor interesse dos pesquisadores psicólogos brasileiros pelo estudo da função lingüística. Em nível internacional, os dois periódicos mais tradicionais da área de neuropsicologia são, segundo Siksou (2005), Cortex e Neuropsychologia, revistas cujos editores pertencem a institutos de Psicologia de universidades inglesas. Isso indica que, internacionalmente, a Psicologia está se dedicando a publicações de neuropsicologia, padrão que tende a ser seguido pelos periódicos brasileiros. Enfatiza-se que talvez não tenha sido encontrado o número esperado de publicações sobre avaliação neuropsicológica da linguagem em periódicos de Psicologia pela limitação dos termos de busca selecionados, como já foi discutido anteriormente.

Além de se esperar maior quantidade de publicações nos periódicos de Psicologia, também se esperava alguma representatividade dos periódicos da área de Lingüística, já que esse campo de conhecimentos também influenciou a área de avaliação neuropsicológica. $\mathrm{O}$ fato de não terem sido encontrados artigos científicos sobre avaliação neuropsicológica da linguagem em revistas de Lingüística pode dever-se a dois fatores: 1) as bases de indexação de artigos consultadas caracterizam-se por apresentarem predominantemente periódicos indexados das áreas da saúde; assim, por exemplo, uma revista bem qualificada como a Cadernos de
Estudos Lingüísticos (Universidade Estadual de Campinas) não foi analisada, e 2) o termo linguagem, utilizado como palavrachave nos procedimentos de busca, é o construto principal da Lingüística, sendo até mesmo redundante colocá-lo como uma palavra do título, do resumo ou das palavras-chave de um estudo publicado; caso subcomponentes da linguagem, tais como nomeação, compreensão, produção, repetição, entre outros, tivessem sido utilizados na presente pesquisa, algumas investigações poderiam ter sido encontradas em periódicos da Lingüística.

À medida que, também no Brasil, a neuropsicologia é considerada uma área interdisciplinar (Rodrigues, 1993), o ideal seria que todas as vertentes das ciências humanas, biológicas, da saúde e da educação envolvidas com a clínica ou com a pesquisa neuropsicológica dedicassem maior espaço para publicações sobre essa área. Destacase a necessidade de publicações sobre o processo de avaliação neuropsicológica, especificamente da função lingüística, tendo em vista os resultados do presente estudo.

Ao se analisar a quantidade de publicações desde 1987, encontraram-se dados promissores. Notou-se um crescimento importante do número de investigações sobre avaliação de linguagem no contexto da neuropsicologia no decorrer dos anos, principalmente desde 2000. Isso ameniza a preocupação frente à quantidade reduzida de publicações encontrada, pois demonstra que está havendo um crescimento exponencial das comunicações científicas sobre o tema em pauta. Dois fatos provavelmente colaboraram para esse crescimento: 1) a década de 1990 foi considerada a década do cérebro, com importantes adventos de técnicas de neuroimagem e de instrumentos 
de avaliação neuropsicológica clínica (Baddeley et al., 2000), e 2) o número de cursos de pós-graduação e de grupos de pesquisa parece estar aumentando nas universidades nacionais. Além disso, pode ter havido um aumento do número de periódicos indexados nos últimos anos.

Quanto à quantidade de publicações nacionais que envolvem instrumentos de avaliação da linguagem relacionadas ao contexto da neuropsicologia e/ou a fins psicométricos, alguns pontos serão discutidos. Primeiramente, considerando-se o total de 187 publicações sobre avaliação de linguagem em geral, entre dissertações, teses e artigos científicos, constatou-se que apenas 57, ou seja, 30,48\% do total baseava-se no uso de algum instrumento. Isso demonstra que, em geral, a função linguagem é examinada com procedimentos não-padronizados de avaliação, como tarefas de nomeação de objetos reais, ou com tarefas experimentais, sem o uso de um instrumento propriamente dito, tal como o Teste Boston para diagnóstico de afasia. $\mathrm{Na}$ quantificação dos estudos neuropsicológicos com instrumento, esse padrão da avaliação de linguagem em geral se repete, já que por volta de $30 \%$ das investigações que abordavam algum instrumento foi considerada neuropsicológica. Na neuropsicologia, tradicionalmente, a avaliação baseia-se em procedimentos não-padronizados ou no uso de tarefas (Alchieri, 2003; Benedet, 1995; Kane, 1991).

Em segundo lugar, complementando essa análise, também foi promovida a quantificação dos estudos que envolviam algum instrumento com e sem fins psicométricos. Foi encontrada uma reduzida quantidade de publicações que apresentava como base para o estudo a preocupação com algum critério psicométrico: apenas 7, de 57 investigações. Especificandose essa quantificação para o contexto neuropsicológico, apenas 2, dos 17 estudos de avaliação neuropsicológica da linguagem que faziam referência a um instrumento, apresentavam fins psicométricos. Esse dado está estreitamente relacionado ao dado anterior, isto é, a tradição de avaliar a linguagem através de procedimentos nãopadronizados no contexto neuropsicológico provavelmente tenha influenciado a demanda de legitimação psicométrica dos instrumentos existentes. Observa-se, no entanto, um forte movimento na neuropsicologia internacional em busca de critérios psicolingüísticos e psicométricos na construção e adaptação de instrumentos neuropsicológicos (Chan, Shum, \& Cheung, 2003; Takahashi, Tanaka, \& Miyaoka, 2006; Thuillard, Colombo, \& Assal, 1992). Tal movimento ainda está insipiente em nível nacional, sendo que a maioria dos estudos psicométricos que envolvem instrumentos de avaliação neuropsicológica da linguagem é publicada em inglês (por exemplo, Mansur, Radanovic, Taquemori, Greco, \& Araújo, 2005).

Prevendo-se maior dedicação da Psicologia à neuropsicologia, provavelmente a influência do movimento da área de avaliação psicológica vai se estender para a avaliação neuropsicológica brasileira. Isso contribuirá muito para a melhoria da qualidade psicométrica dos instrumentos neuropsicológicos, além da qualidade psicolingüística já tradicionalmente considerada.

No cerne do reduzido número de estudos com fins psicométricos sobre a avaliação neuropsicológica da linguagem com instrumentos, está a relação entre a neuropsicologia e a psicometria. Ardila e 
Ostrosky-Solís (1993) abordam essa relação dando ênfase às diferenças existentes entre os objetivos da psicometria e da neuropsicologia. Para os autores, essas duas áreas de estudo vêm desenvolvendo enfoques independentes de avaliação de problemas clínicos. Embora alguns testes sejam utilizados em comum pelas avaliações psicométrica e neuropsicológica, os pressupostos e técnicas são distintos. $\mathrm{Na}$ exploração psicométrica, há uma tendência de se avaliar habilidades cognitivas ou outros construtos psicológicos através de baterias padronizadas, considerandose, predominantemente, os desvios quantitativos de execução. Em contrapartida, na exploração neuropsicológica, há uma tradição de se desenvolver tarefas que possibilitem uma avaliação qualitativa e quantitativa do desempenho de indivíduos normais ou lesados em determinadas habilidades cognitivas, isso porque nem sempre o uso de uma bateria padronizada de subtestes é suficiente para que um diagnóstico neuropsicológico adequado seja realizado (Alchieri, 2003).

As diferenças acima abordadas podem ser sintetizadas nas duas abordagens citadas por Hebben e Milberg (2002). No estudo da avaliação neuropsicológica, há duas abordagens: a quantitativa e a qualitativa. A partir da primeira, baterias como a bateria neuropsicológica de Halstead-Reitan foram formuladas, com padrões psicométricos cuidadosos. De acordo com a segunda, observações organizadas conforme teorias do funcionamento cerebral foram elaboradas e geraram tarefas, tais como as seqüências de observações organizadas por Luria.

Tal tradição de diferenças parece, porém, estar diminuindo. Em obras internacionais de avaliação neuropsicológica, capítulos inteiros estão sendo dedicados à importância da psicometria. Desse modo, a metodologia neuropsicométrica tem sido salientada, com a descrição de procedimentos para a obtenção de normas, fidedignidade e validade (Braun, 1997; Strauss et al., 2006). Além disso, diversos instrumentos de avaliação neuropsicológica geral e avaliação neuropsicológica de linguagem são apresentados em obras clássicas da neuropsicologia, com a apresentação de dados psicométricos. Mencionam-se como exemplos os instrumentos de avaliação de linguagem Token Test, Boston Diagnostic Aphasia Examination e Boston Naming Test (Lezak et al., 2004; Strausset al., 2006).

No que diz respeito aos instrumentos de avaliação neuropsicológica da linguagem encontrados nas pesquisas nacionais consultadas no presente estudo, os mais abordados foram os seguintes: Teste de Nomeação de Boston, Teste Boston para Diagnóstico de Afasia, Teste de Fluência Verbal - Categoria Animais, Teste de Vocabulário por Imagens Peabody e Teste Token. Tais instrumentos são tradicionais na avaliação da função lingüística no contexto neuropsicológico em nível internacional, principalmente no processo diagnóstico dos distúrbios afásicos. O Teste Peabody foi o único não elaborado especificamente para fins neuropsicológicos (Lezak, et al., 2004; Strauss, et al., 2006). Entretanto, no Brasil, pela quantidade de estudos que os mencionaram, observa-se que ainda são pouco difundidos na comunidade científica, ainda sem comercialização disponível para todo o País.

Essa questão remete à falta de instrumentos adaptados para nossa realidade, que corresponde a um desafio a ser solucionado pela pesquisa nacional em neuropsicologia. 
Sabe-se que o uso de instrumentos estrangeiros apenas traduzidos para o português na variedade brasileira não é suficiente (Radanovic \& Mansur, 2002; Serafini, Fonseca, Bandeira, \& Parente, 2005; Villaseñor, Lopez Carlos, Curiel, Medrano, \& Rodríguez, 2003). Torna-se necessária uma adaptação de critérios psicolingüísticos e psicométricos para a população local, para quem a avaliação neuropsicológica contribuirá no processo diagnóstico.

\section{Conclusão}

Com o presente estudo, foi possível obter um panorama geral das pesquisas nacionais que envolvem a avaliação de linguagem no contexto da neuropsicologia. Foram encontrados os seguintes dados:

- número reduzido de pesquisas com foco na avaliação de linguagem no contexto da neuropsicologia;

- concentração das publicações específicas sobre avaliação neuropsicológica da linguagem em periódicos de áreas médicas;

- crescimento de artigos científicos sobre avaliação de linguagem no contexto neuropsicológico, predominantemente nos últimos seis anos;
- pequena quantidade de publicações na área de avaliação neuropsicológica de linguagem que faziam referência a algum tipo de instrumento, assim como de investigações com finalidade psicométrica;

- instrumentos mais estudados em pesquisas sobre avaliação neuropsicológica de linguagem: o Teste de Nomeação de Boston, o Teste de Fluência Verbal Semântica Categoria Animais, o Teste de Vocabulário por Imagens Peabody e o Teste Token.

A partir desses dados, evidencia-se a necessidade de se aumentar a quantidade de pesquisas sobre avaliação neuropsicológica da função lingüística no Brasil. A tradição do uso de tarefas deve continuar sendo perpetuada, com a complementação de instrumentos padronizados com procedimentos psicométricos, visando à qualidade da avaliação do processo relativo ao produto do funcionamento cognitivo lingüístico. Sugere-se, para a continuidade e ampliação do presente estudo, que sejam promovidas revisões sistemáticas da literatura com palavras-chave mais específicas dentro da temática linguagem assim como uma busca na lista de referências citadas nos artigos sobre avaliação neuropsicológica de linguagem e nos currículos dos pesquisadores da área. 
Adriana Jung Serafini - Psicóloga. Especialista em Avaliação Psicológica (UFRGS). Doutoranda em Psicologia (UFRGS e Universidade de Montana). E-mail: adrianaserafini@terra.com.br

Rochele Paz Fonseca - Psicóloga. Fonoaudióloga. Doutora em do Desenvolvimento (UFRGS e Universidade de Montreal). Professora Adjunto da Faculdade de Psicologia e do Programa de Pós-Graduação em Psicologia - ênfase Cognição Humana - da PUCRS. Coordenadora do Grupo de Pesquisa Neuropsicologia Clínica e Experimental. E-mail: rochele.fonseca@gmail.com

Denise Ruschel Bandeira- Psicóloga. Doutora em Psicologia. Professor Adjunto da Graduação em Psicologia e da Pós-graduação em Psicologia, Instituto de Psicologia (UFRGS). E-mail: drbandei@terra.com.br

Maria Alice de Mattos Pimenta Parente - Fonoaudióloga. Doutora em Psicologia. Professor Adjunto da Graduação em Psicologia e da Pós-graduação em Psicologia, Instituto de Psicologia (UFRGS). Coordenadora do Laboratório de Neuropsicolingüística - UFRGS

E-mail: malicemp@terra.com.br

Endereço para correspondência:

Rua Cônego Viana, 247/301, Bairro Rio Branco Cep: 90420-170, Porto Alegre-RS

Recebido 17/11/06 Reformulado 09/05/07 Aprovado 26/05/07

\section{Referências}

Alajouanine, T., \& Lhermitte, F. (1960). Les troubles des activités expressives du language dans l'aphasie. Revue Neurologique, Issy-Les-Moulineaux, 102, 66-91.

Alajouanine, T., Ombredane, A., \& Durand, M. (1939). Le syndrome de désintégration phonétique dans I'aphasie. Paris: Masson.

Alchieri, J. C. (2003). Produção científica brasileira em neuropsicologia: análise de artigos publicados de 1930 a 1999. PSIC: Revista de Psicologia da Vetor Editora, São Paulo, 4(1), 6-13.

Andrade, V. M. Das bases históricas da neuropsicologia à avaliação neuropsicológica. In R. M. Cruz, J. C. Alchieri \& J. R. Sarda (2002), Avaliação e medidas psicológicas (pp. 27-44). São Paulo: Casa do Psicólogo.

Ardila, A., \& Ostrosky-Solís, F. (1993). Diagnóstico del daño cerebral - enfoque neuropsicológico. México: Trillas.

Argimon, I. L., Timm, L. A., Rigoni, M. S., \& Oliveira, M. S. (julho/ dezembro, 2005). Instrumentos de avaliação de memória em idosos: uma revisão. RBCEH: Revista Brasileira de Ciências do Envelhecimento Humano, Passo Fundo, 2(2), 28-35.

Baddeley, A., Bueno, O., Cahill, L., Fuster, J. M., Izquierdo, I., Mcgaugh, J. L., \& Benedet, M. J. (1995). Origen y evolución de la neuropsicología y de sus aportaciones al daño cerebral traumático. In M. J. Benedet, Daño cerebral traumático, neuropsicología y calidad de vida (pp. 117-144). Madrid: Fundación MAPFRE Medicina.

Banco de Teses - CAPES. Brasília: Coordenação de Aperfeiçoamento de Pessoal de Nível Superior. Acesso: http://servicos.capes.gov.br/capesdw/

Biblioteca Digital de Teses e Dissertações - IBICT. Brasília: Instituto Brasileiro de Informação em Ciência e Tecnologia. Acesso: http://bdtd.ibict.br/bdtd

Braun, C. M. J. (1997). Évaluation neuropsychologique. Montréal: Décarie Éditeur.

Brookshire, R. H. (2003). Introduction to neurogenic communication disorders. Missouri: Mosby.

Casanova. (1987). Proceedings of VII Congreso Nacional de Neurología. Barcelona: Sociedad Española de Neurología.
Chan, A. S., Shum, D., \& Cheung, R.W.Y. (2003). Recent development of cognitive and neuropsychological assessment in Asian Countries. Psychological Assessment, Washington, DC, 15(3), 257-267.

Cicerone, K. D., Dahlberg, C., Malec, J. F., Langenbahn, D. M., Felicetti, T., Kenipp, S. et al. (2005). Evidence-based cognitive rehabilitation: Updated review of the literature from 1998 through 2002. Archives of Physical Medicine and Rehabilitation, Indianapolis, 86(8), 1681-1692.

Conselho Federal de Psicologia. (2004). Resolução no 2/2004.

Darvesh, S., Leach, L., Black, S. E., Kaplan, E., \& Freedman, M. (2005). The behavioural neurology assessment. Canadian Journal of Neurological Sciences, Calgary, 32(2), 167-77.

Gil, R. Introduction historique à la neuropsychologie clinique et à la neurologie du comportement. In T. Botez-Marquard \& F. Boller (2005), Neuropsychologie clinique et neurologie du comportement (pp. 19-22). Montreal: Les Presses de I'Université de Montréal.

González, H. M., Mungas, D., \& Haan, M. N. (2005). A semantic verbal fluency test for English-and Spanish-speaking older Mexican-Americans. Archives of Clinical Neuropsychology, Denver, 20(2), 199-208.

Harley, T. (2001). The psychology of language. New York: Psychology Press.

Hebben, N., \& Milberg, W. (2002). Essentials of neuropsychological assessment. New York: John Wiley \& Sons.

Index Psi Periódicos Técnico-Científicos. Brasília: Conselho Federal de Psicologia. Disponível na Biblioteca Virtual em Saúde Psicologia (BVS-Psi). Acesso: http://www.bvs-psi.org.br/

Index Psi Teses. Brasília: Conselho Federal de Psicologia. Disponível na Biblioteca Virtual em Saúde - Psicologia (BVSPsi). Acesso: http://www.bvs-psi.org.br/

Kalbe, E., Reinhold, N., Brand, M., Markowitsch, H. J., \& Kessler, J. (2005). A new test battery to assess aphasic disturbances and associated cognitive dysfunctions - German normative data on the aphasia check list. Journal of Clinical and Experimental Neuropsychology, London, 27(7), 799-794.

Kane, R. L. (1191). Standardized and flexible batteries in neuropsychology: An assessment update. Neuropsychology Review, Amsterdam, 2(4), 281-339. 
Larson, E., Kirschner, K., Bode, R., Heinemanm, A., \& Goodman, R. (2005). Construct and predictive validity of the repeatable battery for the assessment of neuropsychological status in the evaluation of stroke patients. Journal of Clinical and Experimental Neuropsychology, London, 27(1), 16-32.

Lezak, M. D., Howieson, D. B., \& Loring, D. W. (2004). Neuropsychological assessment. New York: Oxford Univertsity Press.

LILACS - Literatura Latino-Americana e do Caribe em Ciências da Saúde. São Paulo: Centro Latino-Americano e do Caribe de Informação em Ciências da Saúde. Disponível na Biblioteca Virtual em Saúde - BIREME. Acesso: http://www.bireme.br

Luria, A. R. (1981). Fundamentos de neuropsicologia. São Paulo: EDUSP.

Mäder, M. J. (2002). Avaliação neuropsicológica: da pesquisa à prática clínica com adultos. In R. M. Cruz, J. C. Alchieri, \& J. R. Sarda (2002), Avaliação e medidas psicológicas (pp. 47-68). São Paulo: Casa do Psicólogo.

Manning, L. (2005). La neuropsychologie clinique. Paris: Armand Colin.

Mansur, L. L., Radanovic, M., Taquemori, L., Greco, L., \& Araújo, G. C. (2005). A study of the abilities in oral language comprehension of the Boston Diagnostic Aphasia Examination - Portuguese version: A reference guide for the Brazilian population. Brazilian Journal of Medical an Biological Research, Ribeirão Preto, 38, 277-292.

Mccormick, L., \& Lezak, M. (2005). L'évaluation neuropsychologique. In T. Botez-Marquard \& F. Boller, Neuropsychologie clinique et neurologie du comportement (pp. 59-70). Montréal: Les Presses de I'Université de Montréal.

Mirsky, A. F. (1989). The neuropsychology of attention: Elements of a complex behavior. In E. Perecman (Ed.), Integrating theory and practice in clinical neuropsycholog (pp. 219-230). Hillsdale, N J: Erlbaum.

Morris, R. G. M., Nadel, L., Routtenberg, A., Xavier, G., \& Da Cunha, C. (2000). The brain decade in debate: I. Neurobiology of learning and memory. Brazilian Journal of Medical and Biological Research, Ribeirão Preto, 33, 993-1002.

Radanovic, M., \& Mansur, L. L. (2002). Performance of a Brazilian population sample in the Boston diagnostic aphasia examination. A pilot study. Brazilian Journal of Medical and Biological Research, Ribeirão Preto, 35, 305-317.
Rao, S. M. (1996). Neuropsychological assessment. In B. S. Fogel, R. B., Schiffer, \& S. M. Rao (Eds.), Neuropsychiatry (pp. 29-45). Oxford: Williamns \& Wilkins.

Rodrigues, N. (1993). Neuropsicologia: uma disciplina científica. In N. Rodrigues \& L. L. Mansur (Eds.), Temas em neuropsicologia (Vol. 1, pp. 1-18). São Paulo: Tec. Art.

SciELO - Scientific Electronic Library Online. São Paulo: Fundação de Amparo à Pesquisa do Estado de São Paulo/Centro LatinoAmericano e do Caribe de Informação em Ciências da Saúde. Acesso: SciELO Brasil, http://www.scielo.br/

Serafini, S., Fonseca, R., Bandeira, D., \& Parente, M. A. (2005). Instrumentos neuropsicológicos de linguagem: panorama nacional e verificação de parâmetros psicométricos no Brasil. Trabalho apresentado no II Congresso Brasileiro de Avaliação Psicológica, Gramado, Brasil, 2005.

Seron, X. (1997). La neuropsychologie cognitive. Paris: PUF.

Siksou, M. (2005). Introduction à la neuropsychologie. Paris: Dunod.

Sohlberg, M. M., \& Mateer, C. A. (2001). Assessment of individuals with cognitive impairments. In M. M. Sohlberg \& C. A. Mateer, Cognitive rehabilitation (pp. 89-122). New York: The Guilford Press.

Strauss, E., Sherman, E. M. S., \& Spreen, O. (2006). A compendium of neuropsychological tests: Administration, norms and commentary. New York: Oxford University Press.

Takahashi, M., Tanaka, K., \& Miyaoka, H. (2006). Reliability and validity of communication skills questionnaire (CSQ). Psychiatry and Clinical Neurosciences, Malden, 60 (2), 211-218.

Thuillard, Colombo, F., \& Assal, G. (1992). Adaptation française du test de dénomination de Boston. Versions abrégées. European Review of Applied Psychology, Nancy, 42(1), 67-73.

Vasconcelos, L. G., Brucki, S. M. D., \& Bueno, O. F. A. (2007). Cognitive and functional dementia assessment tools. Dementia \& Neuropsycholohia, São Paulo, 1(1), 18-23.

Villaseñor, T., Lopez Carlos, E., Curiel, G. R., Medrano, M. A., \& Rodríguez, R. P. (2003). Validación de instrumentos cognitivos en población infantil en niños mexicanos: estudio preliminar. Trabalho apresentado no VIII Congresso da Sociedade Latinoamericana de Neuropsicologia, Montreal, Canadá. 